\title{
ORIGINAL ARTICLE \\ Dietary stress does not strengthen selection against single deleterious mutations in Drosophila melanogaster
}

\begin{abstract}
K MacLellan ${ }^{1}$, L Kwan ${ }^{1,3}$, MC Whitlock ${ }^{2}$ and HD Rundle ${ }^{1}$
Stress is generally thought to increase the strength of selection, although empirical results are mixed and general conclusions are difficult because data are limited. Here we compare the fitness effects of nine independent recessive mutations in Drosophila melanogaster in a high- and low-dietary-stress environment, estimating the strength of selection on these mutations arising from both a competitive measure of male reproductive success and productivity (female fecundity and the subsequent survival to adulthood of her offspring). The effect of stress on male reproductive success has not been addressed previously for individual loci and is of particular interest with respect to the alignment of natural and sexual selection. Our results do not support the hypothesis that stress increases the efficacy of selection arising from either fitness component. Results concerning the alignment of natural and sexual selection were mixed, although data are limited. In the low-stress environment, selection on mating success and productivity were concordant for five of nine mutations (four out of four when restricted to those with significant or near-significant productivity effects), whereas in the high-stress environment, selection aligned for seven of nine mutations (two out of two when restricted to those having significant productivity effects). General conclusions as to the effects of stress on the strength of selection and the alignment of natural and sexual selection await data from additional mutations, fitness components and stressors.
\end{abstract}

Heredity (2012) 108, 203-210; doi:10.1038/hdy.2011.60; published online 27 July 2011

Keywords: deleterious mutations; fitness; mating success; mutation load; sexual selection; stress

\section{INTRODUCTION}

Environmental stress, defined as conditions that reduce absolute fitness relative to that in some other (that is, benign) context (Hoffmann and Hercus, 2000; Martin and Lenormand, 2006; Agrawal and Whitlock, 2010), is pervasive in nature, arising from diverse changes in the biotic and abiotic conditions experienced by an organism. The effects of such stresses on selection have a number of potentially important consequences. If stress commonly increases the strength of selection, for example, adaptation may occur more rapidly than otherwise expected, allowing populations to better colonize novel environments or persist during times of environmental change. If selection is stronger under stress, deleterious mutations may also be more effectively purged, reducing mutation load and inbreeding depression as compared with that under benign conditions.

The conventional view is that selection is stronger on average under more stressful conditions (for example, Parsons, 1987; Uyenoyama, 1993; Kondrashov and Houle, 1994; Jasnos et al., 2008; reviewed by Agrawal and Whitlock (2010)). This may have arisen in part from a tendency to erroneously equate reductions in absolute and relative fitness. However, it also likely reflects a general notion that organisms possess an excess capacity to maintain their performance such that individuals in good condition, for example, because they have experienced a relatively benign environment, are less likely to manifest the effects of a deleterious mutation than individuals that have experienced a more stressful environment and are thus in poor condition. Nonetheless, as highlighted in a recent review (Agrawal and Whitlock, 2010), other conceptual frameworks exist that do not suggest a consistent strengthening of selection under stress, but rather predict variable effects, with stress increasing, decreasing or having no effect on selection depending on the particular circumstances. For example, models of fitness as a flux through a pathway or network suggest that environmental stress, by altering the rate-limiting step in the process, may strengthen or weaken selection on a particular mutation depending on where the effect of this mutation occurs in the pathway (Szathmary, 1993; Keightley, 1996; Segre et al., 2005; Sanjuan and Nebot, 2008). Recent theoretical models of fitness landscapes also fail to support the notion that stress, as represented by a change in environment, will cause a consistent strengthening of selection (Martin and Lenormand, 2006).

A number of studies have compared the fitness effects of individual mutations in high- and low-stress environments in Drosophila (Lewis, 1954; Kondrashov and Houle, 1994; Yang et al., 2001; Fry and Heinsohn, 2002; Wang et al., 2009; Young et al., 2009) and a variety of other organisms (for example, Kishony and Leibler, 2003; Jasnos et al., 2008; for a comprehensive review see Agrawal and Whitlock, 2010). On the whole, these data do not support the conventional view that stress tends to increase the strength of selection. Rather, effects are highly variable, with stress weakening selection almost as often as it strengthens it (Agrawal and Whitlock, 2010). Understanding this heterogeneity of responses will require moving beyond the expectation of a simple, general effect of all stresses to address more specific questions, for instance whether certain classes of stress have particular

${ }^{1}$ Department of Biology, Centre for Advanced Research in Environmental Genomics, University of Ottawa, Ottawa, Ontario, Canada and ${ }^{2}$ Department of Zoology, University of British Columbia, Vancouver, British Columbia, Canada

${ }^{3}$ Current address: Department of Ecology and Evolutionary Biology, University of Toronto, Toronto, Ontario, Canada M5S 3B2.

Correspondence: Dr HD Rundle, Department of Biology, University of Ottawa, 30 Marie-Curie Priv, Ottawa, Ontario, Canada K1N6N5.

E-mail: hrundle@uottawa.ca

Received 14 March 2011; revised 25 May 2011; accepted 31 May 2011; published online 27 July 2011 
effects or tend to impact certain components of fitness. In the former case, for example, there is some indication that stress arising specifically from increased resource competition may strengthen selection on average (Agrawal and Whitlock, 2010).

Our ability to draw detailed and robust conclusions is currently hampered by insufficient data. The effects of stressful environments on particular fitness components, for example male reproductive success, have not been addressed, and data are lacking as to whether stress differentially affects different components. The latter is of particular interest with respect to questions concerning the alignment of natural and sexual selection, and hence whether sexual selection tends to promote adaptation. Males often invest substantial resources in maximizing their reproductive success such that, as for other major components of fitness, the underlying traits affecting it are likely to be condition-dependent (Cotton et al., 2004). Given that much of the genome is likely to contribute to an individual's condition (Houle, 1991; Rowe and Houle, 1996), mutations harmful to condition, and hence deleterious with respect to natural selection, may also tend to reduce male reproductive success (Whitlock and Agrawal, 2009). Sexual selection will therefore also act against such mutations, aligning itself with natural selection to increase population mean fitness. Few data directly address this alignment, however, and the extent to which this may change with environment has received limited attention. In particular, if selection varies with stress, the degree to which natural and sexual selection align in novel or changing environments will depend on the effects of stress on sexual versus non-sexual fitness.

Here, for nine independent, recessive mutations with visible phenotypic effects, we estimate the strength of selection arising from two separate fitness components: male mating success and productivity. Using a laboratory population of Drosophila melanogaster, we quantify how selection on these mutations changes in a benign and a stressful environment, and the degree to which natural and sexual selection align for these mutations in each environment. We estimate male mating success through competitive, multiple-choice mating trials and our productivity assay compares the number of adult offspring produced by mutant and wild-type (stock) females that were raised together in a common micro-environment and then mated to standard males. Replicating across nine independent mutations permits direct tests of the hypotheses of interest that treat separate mutations as replicates, thereby providing general insight into this class of mutations.

\section{MATERIALS AND METHODS}

\section{Study populations}

A laboratory stock population of D. melanogaster was created from a sample of approximately 200 flies collected around Dundas, Ontario, by R Dukas (McMaster University, Canada) in 2006 and 2007, and has been maintained in the laboratory at a large population size since (MacLellan et al., 2009). A large sample of this stock was transferred to the University of Ottawa, Canada, in February 2007 and was maintained in 16 half-pint bottles under constant conditions $\left(25^{\circ} \mathrm{C}, 50 \%\right.$ relative humidity, $12 \mathrm{~L}: 12 \mathrm{D}$ photoperiod) with discreet non-overlapping generations on a standard cornmeal-based food for a minimum of 27 generations prior to the start of the experiment.

Nine populations fixed for different recessive mutations with visible phenotypic effect in adult flies were obtained from the Bloomington stock centre in late 2007 (Table 1) and separately introgressed into the stock. Three of these mutations are X-linked (yellow, forked, white) and six are autosomal (brown, claret, cinnabar, eyeless, plexus, sepia), with six affecting eye phenotype (brown, white, cinnabar, claret, sepia, eyeless), one affecting wing phenotype (plexus) and one each affecting bristles (forked) and body color (yellow). Each mutation was crossed separately into the stock population through five rounds of backcrossing, yielding nine populations that shared a similar outbred stock genetic background but each fixed for a different visible mutation (MacLellan et al., 2009). At least one round of introgression was initiated by a mating in each direction (that is, stock female $\times$ mutant male and mutant female $\times$ stock male) to ensure that the final introgressed mutant populations carried mtDNA and $\mathrm{Y}$ chromosomes from the stock. For each mutation, introgression was begun by crossing 100 stock females with 50-100 mutant males. All subsequent crosses throughout the entire introgression procedure involved 100 individuals of each sex, with a similar number of mutant individuals of each sex collected from the offspring of the final round to found the new introgressed mutant stock. After introgression, mutant populations were maintained under the same conditions as the stock $\left(25^{\circ} \mathrm{C}, 50 \%\right.$ relative humidity, $12 \mathrm{~L}$ : $12 \mathrm{D}$ photoperiod) with discreet non-overlapping generations on a standard cornmeal-based food.

\section{Environmental stress assay}

Environmental stress was imposed through a dietary manipulation that used two different larval foods: the standard laboratory cornmeal-based food to which the population is adapted and a novel corn-flour-based food (see Rundle et al., 2005 for the corn-flour media recipe). To confirm that the novel cornflour food increased stress relative to the standard diet, the egg-to-adult survival and emergence time of individuals from the stock population was compared between the two larval foods. A paired design was employed in which 120 eggs were collected from a single group of stock females and then split in half and immediately transferred to two vials (that is, 60/eggs vial), one containing the standard cornmeal food and the other containing the novel corn-flour food.

Table 1 Effects of nine separate mutations on the average proportion of matings achieved by mutant relative to stock males ( $\left.\bar{p}_{m s}\right)$ and the resulting selective coefficient ( $s_{\mathrm{ms}}$; a negative value corresponds to a deleterious allele) when assayed in two different environments

\begin{tabular}{|c|c|c|c|c|c|c|}
\hline \multirow[t]{2}{*}{ Mutation } & \multicolumn{3}{|c|}{ Ancestral (cornmeal) environment } & \multicolumn{3}{|c|}{ Novel (corn-flour) environment } \\
\hline & $\bar{p}_{\mathrm{ms}}$ & $\mathrm{s}_{m s}$ & $\mathrm{P}$ & $\bar{p}_{\mathrm{ms}}$ & $\mathrm{s}_{m s}$ & $\mathrm{P}$ \\
\hline brown & 0.335 & -0.496 & $<0.001^{*}$ & 0.360 & -0.438 & $<0.001^{*}$ \\
\hline cinnabar & 0.385 & -0.374 & $0.008^{*}$ & 0.340 & -0.485 & $<0.001^{*}$ \\
\hline claret & 0.425 & -0.261 & $0.003^{*}$ & 0.465 & -0.131 & 0.376 \\
\hline eyeless & 0.285 & -0.601 & $<0.001^{*}$ & 0.215 & -0.726 & $<0.001^{*}$ \\
\hline forked & 0.440 & -0.214 & 0.131 & 0.380 & -0.387 & $0.001^{*}$ \\
\hline plexus & 0.480 & -0.0769 & 0.599 & 0.530 & 0.128 & 0.368 \\
\hline sepia & 0.435 & -0.230 & $0.044^{*}$ & 0.470 & -0.113 & 0.390 \\
\hline white & 0.140 & -0.837 & $<0.001^{*}$ & 0.105 & -0.883 & $<0.001^{*}$ \\
\hline yellow & 0.365 & -0.425 & $0.002^{*}$ & 0.440 & -0.214 & 0.131 \\
\hline Mean ( \pm s.e) & $0.366(0.034)$ & $-0.391(0.077)$ & & $0.367(0.045)$ & $-0.361(0.106)$ & \\
\hline
\end{tabular}

Significance $(P)$ was evaluated by one-sample $t$-test $(t)$ to determine whether $\bar{p}_{\mathrm{ms}}$ differed from 0.5 (the null hypothesis of equal mating success of mutant and stock males), treating cages as replicates ( $\mathrm{df}=19$ in all cases). The asterisks denote $P \leqslant 0.05$. 
One hundred replicate pairs were set up over a 72-h period and the eggs for each replicate were collected from a unique set of females. The total number of adult offspring and the timing of emergence (to the nearest $24 \mathrm{~h}$ ) were recorded for each vial until all offspring had emerged. The effect of larval rearing environment on the total number of adults emerging, and their timing of emergence, were tested separately using paired $t$-tests (two-tailed).

\section{Male mating success assay}

For each mutation, the mating success of mutant relative to stock males was determined by using a multiple-choice mating assay separately for males raised in each of the two larval food environments. Each replicate involved 20 stock females placed together in a translucent plastic cage $(14 \times 14 \times 14 \mathrm{~cm})$ with 20 mutant and 20 stock males. The cages were shielded from sight to minimize disturbance and mating pairs were removed by aspiration at approximately 10-min intervals. To ensure that females had a choice between males of both types, only the first 10 mating pairs were collected from any single cage, after which the cage was terminated and the remaining individuals were discarded. Males from the mating pairs were identified as either stock or mutant by phenotype.

Forty replicate cages were performed for each mutant population, 20 in which males were raised in the standard cornmeal food environment and 20 in which they were raised for a single generation in the novel corn-flour food environment. Females were raised on the standard cornmeal food in all cases such that differences in the mating success of mutant relative to stock males in the two environments could be attributed to changes in the males and not the females. Individuals for use in the mating trials were collected as virgins upon emergence by using light $\mathrm{CO}_{2}$ anesthesia and held separately by sex (females in groups of 10, males in groups of five) in vials containing $5 \mathrm{ml}$ of their respective food for 5 days prior to the assay. The assay was performed in blocks such that 1-3 mutant populations were tested within a single environment during any particular generation.

The mating success of mutant relative to stock males was calculated as a proportion $\left(p_{\mathrm{ms}}\right)$ by dividing the number of mutant matings $\left(x_{\mathrm{m}}\right)$ by the sum of the number of mutant and stock $\left(x_{\mathrm{s}}\right)$ matings within a cage (that is, $p_{\mathrm{ms}}=x_{\mathrm{m}}$ / $\left.\left(x_{\mathrm{m}}+x_{\mathrm{s}}\right)\right)$. The effect of each individual mutation on male mating success was evaluated by using a one-sample $t$-test (two-tailed), treating population cages as replicates, to determine whether the success of mutant males differed significantly from 0.5 (that is, the null hypothesis of equal mating success of mutant and stock males). To determine the effect of these mutations overall, the mating success of each mutant population was then calculated as the average proportion across all 20 replicate cages of that mutation in a particular environment $\left(\bar{p}_{\mathrm{ms}}\right)$. A one-sample (two-tailed) $t$-test, treating mutations as replicates, was then used to determine whether these values differed significantly from 0.5. None of the above results changed qualitatively when proportions were arcsine-square root-transformed prior to the analyses, so results of the untransformed analyses are presented for simplicity. Selection coefficients describing the homozygous effects of each mutation on mating success in each environment $\left(s_{\mathrm{ms}}\right)$ were calculated by defining the fitness of the mutant as $1+s_{\mathrm{ms}}$ times the fitness of the stock (that is, $\left.x_{\mathrm{m}}=\left(1+s_{\mathrm{ms}}\right) x_{\mathrm{s}}\right)$ such that $s_{\mathrm{ms}}=\left(x_{\mathrm{m}} / x_{\mathrm{s}}\right)-1$. Selection coefficients were therefore estimated as: $s_{\mathrm{ms}}=\left(2 \bar{p}_{\mathrm{ms}}-1\right) /\left(1-\bar{p}_{\mathrm{ms}}\right)$. A paired $t$-test (two-tailed) was used to determine whether the strength of selection differed overall between the two environments treating mutations as replicates.

\section{Productivity assay}

Productivity was determined by counting the total number of adult offspring that emerged from eggs laid by a single female in a $24-\mathrm{h}$ period when mated to a randomly chosen stock male. Productivity therefore represents a combined measure, in an environment, of the fecundity of a particular female and the egg-to-adult survivorship of her male and female offspring. Replicate females were raised in the ancestral (cornmeal) and novel (corn-flour) food environments and laid eggs in the same environment in which they were raised. Productivity was measured in blocks such that females from two mutant populations and the stock were assayed in both environments within any particular generation.
To control for replicate-specific environmental effects on productivity, the two mutant and the stock populations assayed within a particular block were raised together in a common micro-environment (that is, vial) for one generation prior to the productivity assay (Figure 1). Within each block, a single non-virgin female (previously mated with one or more of her own males) from each of two mutant populations and the stock were placed together in a single vial for $24 \mathrm{~h}$ of egg laying, after which they were discarded. Approximately 150 of such 'mixed' vials were created for each of the two environments (cornmeal and corn-flour larval media). Four female offspring were collected as virgins upon emergence from a particular mixed vial using light $\mathrm{CO}_{2}$ anesthesia: two stock females and one female of each of the two mutants. These females were held individually in vials containing $10 \mathrm{ml}$ of their respective food (that is, cornmeal or corn-flour) for 5 days. Four virgin males were then collected from a single vial of stock flies, raised on cornmeal food, and a single stock male was added to each of the four vials. The use of standard stock males controls for any differential effects of males on female productivity. Owing to handling errors and the death of some flies, an average of 126 replicate vials were set up for each combination of mutation and environment (range 112-153), with each vial containing $10 \mathrm{ml}$ of the environmentappropriate food (cornmeal or corn-flour-based). After $24 \mathrm{~h}$, the male and female were discarded and all offspring subsequently emerging from each vial were counted.

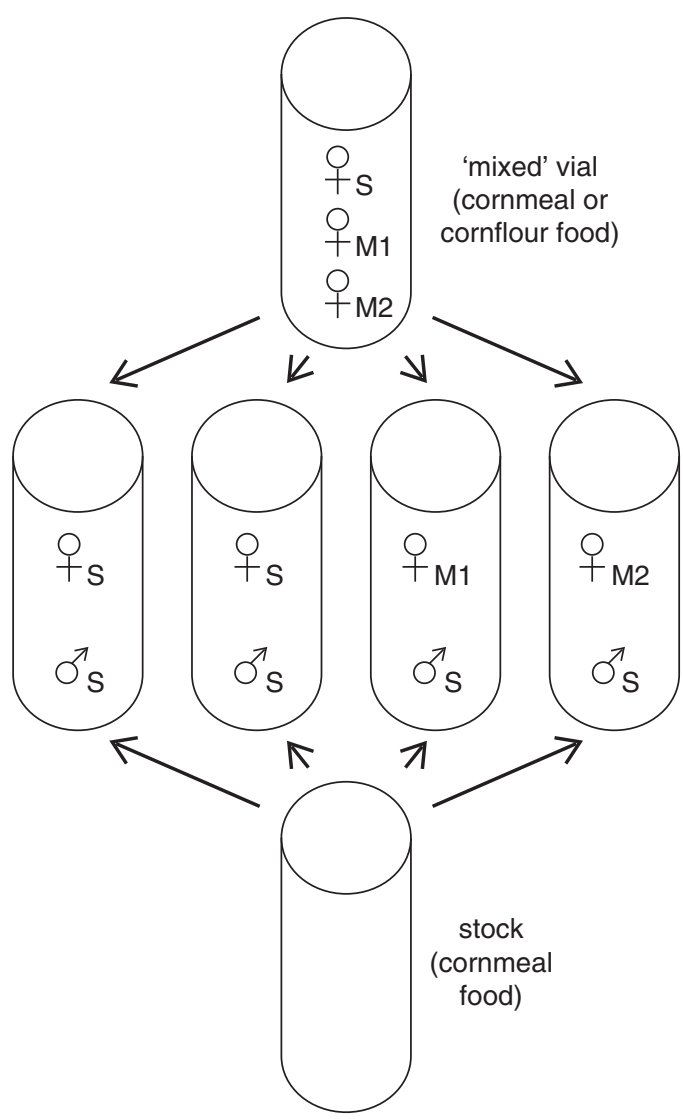

Figure 1 Overview of the design of the productivity assay. A single female from each of the stock and two of the mutant populations were mated to their own males and then placed together for oviposition in a single 'mixed' vial containing either the ancestral (cornmeal) or the novel (corn-flour) food. From this vial, four virgin females were collected, two stock and one of each of the two mutants, and used to set up four productivity vials containing the same food as that on which the female was raised. To each vial was added a single stock male, raised in cornmeal, with all four males collected from the same vial. Approximately 150 replicates of this design were set up for each pair of mutants. 
The effect of each individual mutation on productivity was evaluated by using 'difference scores' calculated as the number of adult offspring produced by a mutant female minus the average number produced by the two stock females raised in the same vial as the mutant (Figure 1). For each mutation, a one-sample $t$-test (two-tailed) was used to determine whether these scores differed significantly from 0 (that is, the null hypothesis of no effect of the mutation on productivity). To test the overall effect of these mutations on productivity, a one-sample (two-tailed) $t$-test, treating mutations as replicates, was used to determine whether the mean difference differed significantly from 0 in each environment. Selection coefficients describing the effects of each mutation on productivity $\left(s_{\mathrm{p}}\right)$ were calculated by comparing the average productivity for each mutation in each environment $\left(\bar{w}_{\mathrm{m}}\right)$ with the average productivity of the stock that was raised in the same set of vials $\left(\bar{w}_{\mathrm{s}}\right)$. The fitness of the mutant was again defined as $1+s_{\mathrm{p}}$ times the fitness of the stock, yielding: $s_{\mathrm{p}}=\frac{\bar{w}_{\mathrm{m}}}{\bar{w}_{\mathrm{s}}}-1$. These selective coefficients represent the combined effects of these mutations on female fecundity when homozygous, and male and female survival (egg to adult emergence) when heterozygous. A paired $t$-test (twotailed) was used to determine whether the strength of selection differed overall between the two environments, treating mutations as replicates.

\section{Statistical analyses}

The productivity and mating success measures for the individual mutations above were approximately normally distributed and, given the robustness of $t$-tests to violations of this assumption, we therefore employ parametric analyses. However, in a handful of cases a normal distribution could be statistically rejected. Although the distributions in these cases were unimodal and roughly symmetrical, at the suggestion of a reviewer we repeated the analyses by using non-parametric Wilcoxon sign ranked tests. While we present these results for completeness, our interpretation focuses on the parametric analyses given our a prior decision that these were appropriate. For the overall tests of the various hypotheses that treated the nine mutations as replicates, none of the results changed qualitatively when non-parametric analyses were employed and we therefore presented the results of the parametric analyses only.

\section{RESULTS}

\section{Effects on mating success and productivity}

In the ancestral environment, the mating success of mutant males was reduced relative to stock males for all nine mutations, seven significantly when considered individually (Table 1). This pattern is significant overall $(t=-3.90, \mathrm{df}=8, P=0.005)$, indicating a general tendency for such mutations to reduce male mating success relative to wild-type males. In the novel environment, eight of the nine mutations reduced male mating success with five of these being significant when considered individually (Table 1). Again, this pattern is significant overall ( $t=-2.96, \mathrm{df}=8, P=0.018$ ), suggesting that such mutations remain deleterious in general in this environment. Only plexus increased male mating success and its effect was non-significant (Table 1). Results of the tests of the individual mutations are not changed qualitatively if non-parametric analyses are used instead, with the single exception that sepia becomes marginally non-significant in the ancestral environment (the $P$-value changes from 0.044 to 0.065 ).

In the ancestral environment, the productivity of mutants was reduced relative to the stock for five of the nine mutations, three significantly and the fourth borderline (white: $P=0.066$ ) when considered individually (Table 2). None of the mutations significantly increased productivity. Across mutations, the mean reduction in mutant productivity was not significant in a two-tailed test ( $t=-1.46, \mathrm{df}=8, P=0.182$ ). In the novel environment, six of the nine mutations decreased productivity relative to the stock, with two of these being significant individually (Table 2). Again, none of the mutations significantly increased productivity. For the novel environment, the mean reduction in mutant productivity overall was not significant in a two-tailed test $(t=-1.58, \mathrm{df}=8, P=0.154)$. Using non-parametric analyses instead, none of the previously significant mutations in either environment become non-significant and two previously non-significant mutations (cinnabar and forked) become significant in the ancestral environment $(P=0.047$ and 0.048 , respectively).

\section{Changes in selection with stress}

The egg-to-adult survival of stock individuals was reduced by $41.6 \%$ on average in the novel corn-flour food environment as compared with the standard food environment, with an average of 28.5 versus 48.8 adults emerging, respectively, from vials created with 60 eggs. This decrease is significant (paired $t$-test, $t=15.7, \mathrm{df}=899, P<0.001$ ) and is very similar to the $42.7 \%$ reduction in average stock productivity observed across the five blocks of the productivity assay (41.3 versus 72.0 adult offspring on average on the novel corn-flour and the standard food, respectively). The emergence time of stock individuals was also increased by 5.1 days on average on the novel corn-flour food as compared with the standard food, a difference that is also significant (paired $t$-test, $t=-48.9, \mathrm{df}=898, P<0.001$ ). These differences in egg-to-adult survival and emergence time indicate that the

Table 2 Effects of nine separate mutations on the productivity of mutant $\left(\bar{w}_{m}\right)$ and stock $\left(\bar{w}_{s}\right)$ females, and the resulting selective coefficient ( $s_{\mathrm{p}}$; a negative value corresponds to a deleterious allele) when assayed in two different environments

\begin{tabular}{|c|c|c|c|c|c|c|c|c|}
\hline \multirow[t]{2}{*}{ Mutation } & \multicolumn{4}{|c|}{ Ancestral (cornmeal) environment } & \multicolumn{4}{|c|}{ Novel (corn-flour) environment } \\
\hline & $\bar{w}_{\mathrm{m}}$ & $\bar{w}_{\mathrm{m}}$ & $\mathrm{s}_{p}$ & $\mathrm{P}$ & $\bar{w}_{\mathrm{m}}$ & $\bar{w}_{\mathrm{m}}$ & $\mathrm{s}_{p}$ & $P$ \\
\hline brown & 79.5 & 76.0 & 0.045 & 0.385 & 50.9 & 53.3 & -0.045 & 0.316 \\
\hline cinnabar & 82.0 & 76.0 & 0.079 & 0.101 & 51.3 & 53.3 & -0.037 & 0.404 \\
\hline claret & 60.7 & 70.6 & -0.140 & $<0.001^{*}$ & 35.1 & 35.0 & 0.001 & 0.991 \\
\hline eyeless & 57.5 & 68.8 & -0.164 & $<0.001^{*}$ & 27.8 & 43.5 & -0.361 & $<0.001 *$ \\
\hline forked & 73.9 & 70.6 & 0.047 & 0.223 & 32.6 & 35.0 & -0.070 & 0.265 \\
\hline plexus & 69.4 & 72.8 & -0.047 & 0.292 & 41.2 & 38.0 & 0.084 & 0.133 \\
\hline sepia & 47.5 & 71.8 & -0.338 & $<0.001^{*}$ & 27.4 & 36.5 & -0.249 & $<0.001$ * \\
\hline white & 63.8 & 71.8 & -0.112 & 0.066 & 33.2 & 36.5 & -0.089 & 0.187 \\
\hline yellow & 74.5 & 72.8 & 0.023 & 0.642 & 41.3 & 38.0 & 0.086 & 0.142 \\
\hline Mean ( \pm s.e) & $67.6(3.7)$ & $72.4(0.8)$ & $-0.067(0.045)$ & & $37.9(3.0)$ & $41.0(2.5)$ & $-0.076(0.049)$ & \\
\hline
\end{tabular}

Significance $(P)$ was evaluated by one-sample $t$-test $(t)$ to determine whether the difference in productivity (mutant-stock) differed from 0 (the null hypothesis of equal productivity of stock and mutant females), treating vials as replicates. Degrees-of-freedom vary depending on the number of replicate vials and range from 111 to 152 . The asterisks denote $P \leqslant 0.05$. 


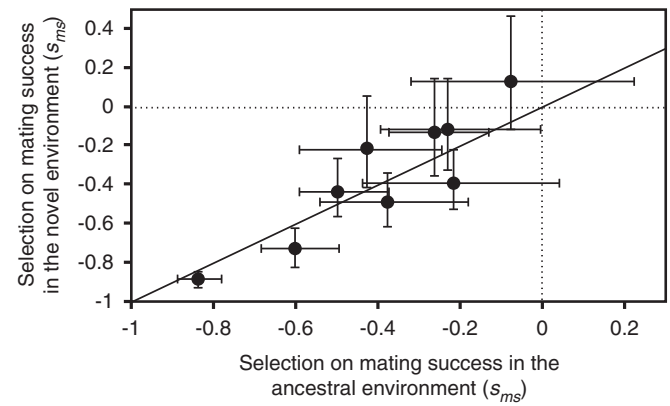

Figure 2 Selection coefficients arising from the effects of nine separate mutations on competitive male mating success when males are raised in their ancestral (cornmeal-based) or a novel and more stressful (corn-flourbased) larval food environment $(r=0.903, P<0.001)$. The error bars represent $95 \%$ confidence intervals from 1000 bootstrap replicates. A 1:1 line is shown for reference.

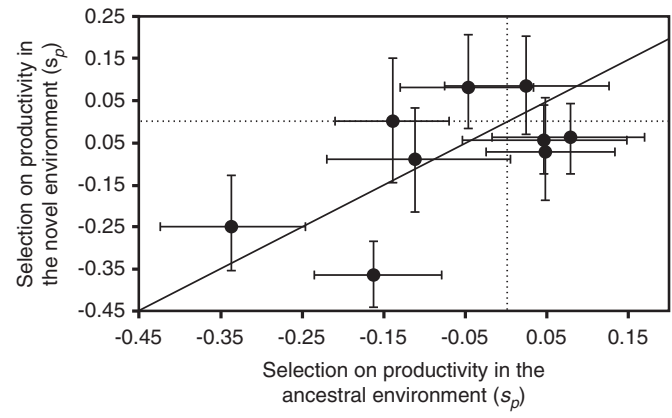

Figure 3 Selection coefficients arising from the effects of nine separate mutations on productivity when females are raised in their ancestral (cornmeal-based) or a novel and more stressful (corn-flour-based) larval food environment $(r=0.615, P=0.078)$. The error bars represent $95 \%$ confidence intervals from 1000 bootstrap replicates. A 1:1 line is shown for reference.

novel corn-flour environment can be considered as more stressful. Both results remain highly significant in non-parametric analyses.

Selective coefficients for these mutations tended to correspond between the two environments, falling roughly along the 1:1 line and generating a cross-environment correlation (estimated as the correlation of mean selective coefficients) that was strong and highly significant for male mating success $(r=0.903, t=5.55, \quad \mathrm{df}=87$, $P<0.0001$; Figure 2) and that, although somewhat weaker, was still substantial and approached significance for productivity $(r=0.615$, $t=2.06, \mathrm{df}=87, P=0.078$; Figure 3 ). Selection coefficients did not differ on average between these two environments for either male mating success (paired $t$-test, $t=0.607, \mathrm{df}=88, P=0.561$ ) or productivity (paired $t$-test, $t=-0.196, \mathrm{df}=88, P=0.849$ ), providing no evidence that stress increased, or otherwise altered, the overall effects of these mutations on these fitness components.

\section{Sexual selection and mutation load}

Support for the alignment of natural and sexual selection was mixed. In the ancestral environment overall, five of the nine mutations had corresponding effects on male mating success and productivity (Figure 4). However, restricting analysis to the three mutations that significantly reduced productivity in this environment (claret, eyeless, sepia), all three caused significant reductions in male mating success

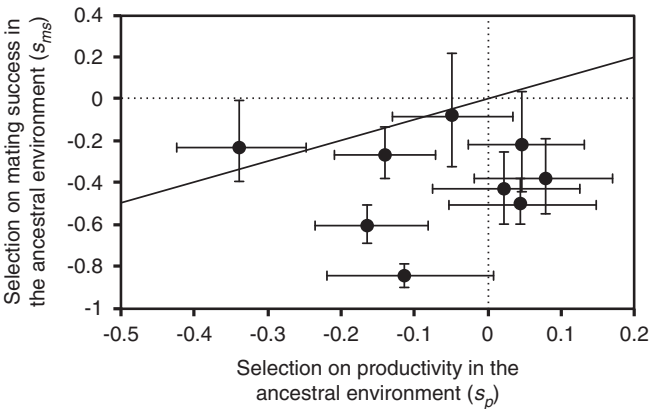

Figure 4 Exploring the alignment of natural and sexual selection for nine separate mutations in the ancestral (cornmeal-based food) environment. Natural and sexual selection coefficients were estimated from the effects of each mutation on productivity and male mating success, respectively. The error bars represent 95\% confidence intervals from 1000 bootstrap replicates. A 1:1 line is shown for reference.

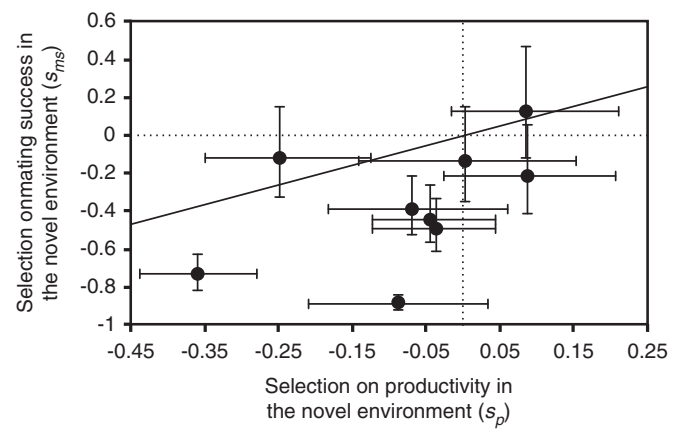

Figure 5 Exploring the alignment of natural and sexual selection for nine separate mutations in the novel (corn-flour-based food) environment. Natural and sexual selection coefficients were estimated from the effects of each mutation on productivity and male mating success, respectively. The error bars represent $95 \%$ confidence intervals from 1000 bootstrap replicates. A $1: 1$ line is shown for reference.

and white, which caused a borderline-significant reduction in productivity ( $P=0.066$ ), also decreased male mating success significantly in this environment. In the novel environment, natural and sexual selection aligned for seven of the nine mutations overall (Figure 5). Only two mutations significantly reduced productivity, and both also reduced male mating success, significantly for one (eyeless) and nonsignificantly for the other (sepia).

\section{DISCUSSION}

In evolutionary biology, it is widely believed that the effects of deleterious alleles are more pronounced in stressful environments (Agrawal and Whitlock, 2010). While this is supported for some alleles (for example, Korona, 1999; Remold and Lenski, 2001; Szafraniec et al., 2001; Fry and Heinsohn, 2002; Baer et al., 2006), for others there is little or no evidence for increased strength of selection with stress (Korona, 1999; Kishony and Leibler, 2003; Baer et al., 2006). The current study provides additional support for the view that stress does not, on average, increase the efficacy of selection. In particular, within the confines of nine mutations, two fitness components and one stressor, despite strong evidence of selection against several deleterious alleles, there was no support for the stress hypothesis. While such additional data are valuable, generalizations as to the average effect of stress on the strength of selection remain hampered by the limited 
range and number of mutations and stressors, and this therefore remains an important topic of investigation.

With respect to mating success, although a number of studies have explored the interaction of stress and mating behavior (Patton and Krebs, 2001; Lidgard et al., 2008) and many have documented a relationship between condition and secondary sexual characteristics (Jennions et al., 2001; Cotton et al., 2004), to our knowledge this study is the first to quantify the effects of stress on selection for single loci. Many studies suggest that sexually selected traits are conditiondependent (Cotton et al., 2004) and this may lead one to conjecture that, under stressful conditions, relatively healthy males should have more preferred secondary sexual characteristics, and thus a higher mating success, than less healthy males. However, the hypothesis that stress leads to stronger selection through mating success for deleterious alleles has been little tested, if at all, and current data suggest that selection for mating success at the level of the locus does not increase in general under stress. Rather, our results suggest that males with deleterious alleles pay no extra price in relative fitness under more stressful as compared with benign conditions. On the other hand, stress was imposed in our experiment through a novel diet that reduced larval survival and increased development time, and the extent to which these effects carried over to the emerging adults is not known. It is therefore possible that the surviving males suffered no ill effects of this larval stress, thereby explaining the strong correspondence between environments in the selective coefficients on these mutations arising from their effects on male mating success. In general, the extent to which the effects of stress experienced during certain life stages extends to others has received limited attention yet could have important implications. General conclusions on the effects of stress on mating success therefore await further data addressing a range of stresses and a variety of mutations.

The mutations used in this study are not a random selection of all possible mutations. Instead, they are mutations that have fairly large, visible phenotypic effects, and the selection coefficients against them tend to be larger than most typical mutations. These mutations may therefore behave differently from other mutations, and it is possible, for example, that their deleterious effects on male mating success may be relatively insensitive to diet as compared with their pleiotropic effects on other traits affecting fecundity and viability, such as resource acquisition. Direct empirical data are lacking with which to address such questions and more work is needed. Nevertheless, having said this, these mutations have large enough effects that changes in the strength of selection ought to be detectable, which may be much more difficult for alleles of smaller effect. The fact that changes in selection with stress were not detectable for even these large-effect mutations argues that the effects of stress are in fact relatively small. However, insufficient power may also have been an important factor in our inability to detect an effect of stress given that selection on these mutations arising from productivity was often not statistically detectable (Table 2).

On the other hand, these mutations are unlikely to be biased from the point of view of the type of function that they have. They are not chosen from known DNA-repair pathways or from kinds of genes that are known to be upregulated during stress like chaperone proteins. As a result, they are unlikely to be of the specific classes of genes that are likely to show increased selection coefficients during stress (Agrawal and Whitlock, 2010). Similarly, the environmental stressor that was used is likely to be common in nature and to affect all individuals equally, unlike density stress that has been hypothesized to create scramble competition and therefore cause more fit individuals to have better access to resources and therefore different environments
(Agrawal and Whitlock, 2010). The fact remains, however, that we have considered only one type of stress imposed through a particular dietary manipulation; data addressing the effects of additional stressors are needed to determine the generality of our results.

Martin and Lenormand (2006) have identified several predictions about the nature of selection in more stressful environments on which the current results may shed some light. First, they predict that alleles should be more variable in their effects in stressful rather than benign environments. In the current study, we find that the variance in fitness among mutations is increased in the stressful environment by about $87 \%$ for male mating success and $18 \%$ for productivity, although these increases in variance in $s$ are not significant (Brown-Forsythe test, $P=0.36$ for male mating success and $P=0.79$ for productivity). Similar results were found recently for a series of 20 mutations with dominant visible effects in $D$. melanogaster, where the variance in viability selection increased by $77 \%(P=0.11)$ in a more stressful environment as compared with a less stressful one (Wang et al., 2009).

Second, Martin and Lenormand (2006) predict that some mutations will switch from deleterious to beneficial under stressful conditions, but we find little support for this. Seven mutations had significant deleterious effects on mating success in the ancestral environment, and four of these (brown, cinnabar, eyeless, white) remained significantly deleterious in the novel environment. The others remained deleterious but not significantly so. Three mutations had significantly deleterious effects on productivity in the ancestral environment, and two of these (eyeless and sepia) remained significantly deleterious in the novel environment. The third (claret) switched sign, but there was no statistical support for saying that it had become beneficial. In all, there is little support from these data for the prediction that some deleterious alleles become beneficial under stress. However, these data do not provide perfect conditions for testing these predictions because these alleles are of relatively large effect. The theory of Martin and Lenormand would predict that smalleffect mutations are more likely to shift from deleterious to beneficial under stress. We have insufficient power to investigate such changes and our data should therefore not be taken as refuting this prediction.

Finally, Wang et al (2009) noted that Martin and Lenormand's theory also predicts that, for unconditionally deleterious mutations (that is, deleterious in both environments), selection should increase under more stressful conditions. For mating success, four mutations are unconditionally deleterious. Selection against three of them (cinnabar, eyeless, white) is stronger in the novel than the ancestral environment, whereas selection against the other (brown) shows the reverse pattern. None of the changes for mating success are statistically significant however (two-tailed $t$-tests treating cages as replicates: brown, $P=0.412$; cinnabar, $P=0.265$; eyeless, $P=0.130$; white, $P=0.188)$. For productivity, two mutations are unconditionally deleterious and the estimate of $s$ is higher in the novel environment as compared with the ancestral environment for one of these (eyeless, $P=0.004$ ) and non-significantly lower for the other (sepia).

If reproductive success is condition-dependent, sexual selection may align with natural selection to promote adaptation and the purging of deleterious mutations. Selection on males in particular has been shown to be potentially important in the amelioration of mutation load because, in polygamous mating systems with low paternal investment, selection on males can reduce the frequency of deleterious alleles without causing a reduction in the mean fitness of the population (Manning, 1984; Kodric-Brown and Brown, 1987; Koeslag and Koeslag, 1993; Whitlock, 2000; Agrawal, 2001; Siller, 2001; Lorch et al., 2003). To date, the effect on male reproductive success has been quantified for 14 different mutations with significant deleterious 
effects on non-sexual fitness (Whitlock and Bourguet, 2000; Pischedda and Chippindale, 2005; Sharp and Agrawal, 2008). Of these 14 mutations, sexual selection on males aligned with natural selection for 10 of them, increasing selection against them (Whitlock and Agrawal, 2009).

In our experiment, effects on male mating success and productivity were concordant for five of nine mutations overall in the ancestral (that is, low stress) environment to which the population is adapted (Figure 4), and seven of nine mutations overall in the novel (that is, high stress) environment (Figure 5). Support for the alignment of natural and sexual selection is therefore weak in this respect and in neither environment can we reject the general absence of an association. While data with which to evaluate this for individual mutations are limited (productivity effects were significant, or nearly so, for only four/two mutations in the ancestral/novel environments, respectively), there is a stronger trend suggesting alignment of natural and sexual selection when the analyses are restricted to this subset. In particular, of the four mutations in the ancestral environment that had significant or near-significant deleterious effects on productivity, male mating success was significantly reduced for all of these (claret, eyeless, sepia, white). Whitlock and Bourguet (2000) independently found the same result for claret. The other three, however, are novel and show concordant effects on mating success and productivity, yielding 13 of 17 independent mutations across studies that support the hypothesis that natural and sexual selection tend to align. In the novel environment, mating success was also reduced for both of the mutations affecting productivity, although this reduction was significant for only one of these (eyeless).

Our choice of mutations is unlikely to be biased with respect to the alignment of natural and sexual selection because they were not chosen with respect to their effects on condition. However, these mutations do have visible phenotypic effects that may make them direct targets of sexual selection. Such direct selection may serve to weaken the alignment of natural and sexual selection that arises from condition-dependent reproductive success. We have also considered the effect of these mutations on mating success only; the condition dependence of other components of male sexual fitness, such as sperm competitive ability or search effort (MacLellan et al., 2009), could also be important. Finally, for sexual selection to reduce mutation load and promote adaptation, total selection in males must be stronger than it is in females (Whitlock and Agrawal, 2009). Unfortunately, as with most of the other studies, we cannot compare selection in males and females directly because our productivity measure included the survival to adulthood of male and female offspring heterozygous for the mutation, although the degree that these mutations are recessive will minimize this problem in this case. More data are needed with which selection can be compared directly between the sexes (for example, Sharp and Agrawal, 2008).

An emerging picture suggests that selection against deleterious mutations is enhanced-usually, but not always — by reduced mating success of males that carry those mutations and that selection is not affected by a stressful environment on average, although for individual genes the effect is variable. To understand natural selection, we need to know not only its effects at the phenotypic level, but we would also like to be able to predict how selection on individual loci will be affected over all fitness components and by interactions with the environment. In attempting to do this, some trends emerge (such as the tendency for alleles to be selected in the same direction owing to their effects on productivity and male mating success) but other patterns are not strong (such as any possible relationship between stress and the strength of selection). Currently, the strongest pattern appears to be one of heterogeneity among loci, and it is therefore only by doing more studies at the level of the single locus that we will better understand the patterns of selection that are possible.

\section{DATA ARCHIVING}

Genotype data have been deposited at Dryad: doi:10.5061/dryad.84g54.

\section{CONFLICT OF INTEREST}

The authors declare no conflict of interest.

\section{ACKNOWLEDGEMENTS}

We thank S Khair, V Rontondo and P Wu for help in the laboratory and four anonymous reviewers for comments on previous versions of the manuscript. $\mathrm{R}$ Dukas provided the stock population of D. melanogaster. This work was funded by a grant to HDR from the Natural Sciences and Engineering Research Council of Canada and an Ontario Early Researcher Award.

Agrawal AF (2001). Sexual selection and the maintenance of sexual reproduction. Nature 411: 692-695.

Agrawal AF, Whitlock MC (2010). Environmental duress and epistasis: how does stress affect the strength of selection on new mutations? Trends Ecol Evol 25: 450-458.

Baer CF, Phillips N, Ostrow D, Avalos A, Blanton D, Boggs A et al. (2006). Cumulative effects of spontaneous mutations for fitness in Caenorhabditis: role of genotype, environment and stress. Genetics 174: 1387-1395.

Cotton S, Fowler K, Pomiankowski A (2004). Do sexual ornaments demonstrate heightened condition-dependent expression as predicted by the handicap hypothesis? Proc Roy Soc London B 271: 771-783.

Fry JD, Heinsohn SL (2002). Environment dependence of mutational parameters for viability in Drosophila melanogaster. Genetics 161: 1155-1167.

Hoffmann AA, Hercus MJ (2000). Environmental stress as an evolutionary force. Bioscience 50: 217-226.

Houle D (1991). Genetic covariance of fitness correlates: what genetic correlations are made of and why it matters. Evolution 45: 630-648.

Jasnos L, Tomala K, Paczesniak D, Korona R (2008). Interactions between stressful environment and gene deletions alleviate the expected average loss of fitness in yeast. Genetics 178: 2105-2111.

Jennions MD, Moller AP, Petrie M (2001). Sexually selected traits and adult survival: a meta-analysis. Q Rev Biol 76: 3-36.

Keightley PD (1996). Metabolic models of selection response. J Theor Biol 182: 311-316.

Kishony R, Leibler S (2003). Environmental stresses can alleviate the average deleterious effect of mutations. J Biol 2: 14.

Kodric-Brown A, Brown JH (1987). Anisogamy, sexual selection, and the evolution and maintenance of sex. Evol Ecol 1: 95-105.

Koeslag JH, Koeslag PD (1993). Evolutionarily stable meiotic sex. J Hered 84: 396-399.

Kondrashov AS, Houle D (1994). Genotype-environment interactions and the estimation of the genomic mutation rate in Drosophila melanogaster. Proc Roy Soc London B 258: 221-227.

Korona R (1999). Genetic load of the yeast Saccharomyces cerevisiae under diverse environmental conditions. Evolution 53: 1966-1971.

Lewis HW (1954). Studies on a melanoma-producing lethal in Drosophila. J Exp Zool 126: 235-275.

Lidgard DC, Boness DJ, Bowen WD, Mcmillan JI (2008). The implications of stress on male mating behavior and success in a sexually dimorphic polygynous mammal, the grey seal. Horm Behav 53: 241-248.

Lorch PD, Proulx S, Rowe L, Day T (2003). Condition-dependent sexual selection can accelerate adaptation. Evol Ecol Res 5: 867-881.

Maclellan K, Whitlock MC, Rundle HD (2009). Sexual selection against deleterious mutations via variable male search success. Biol Lett 5: 795-797.

Manning JT (1984). Males and the advantage of sex. J Theor Biol 108: 215-220.

Martin G, Lenormand T (2006). The fitness effect of mutations across environments: a survey in light of fitness landscape models. Evolution 60: 2413-2427.

Parsons PA (1987). Evolutionary rates under environmental stress. Evol Bio/ 21: 311-347.

Patton ZJ, Krebs RA (2001). The effect of thermal stress on the mating behavior of three Drosophila species. Physiol Biochem Zool 74: 783-788.

Pischedda A, Chippindale A (2005). Sex, mutation and fitness: asymmetric costs and routes to recovery through compensatory evolution. J Evol Biol 18: 1115-1122.

Remold SK, Lenski RE (2001). Contribution of individual random mutations to genotypeby-environment interactions in Escherichia coli. Proc Natl Acad Sci USA 98: $11388-11393$.

Rowe L, Houle D (1996). The lek paradox and the capture of genetic variance by condition dependent traits. Proc Roy Soc London B 263: 1415-1421.

Rundle HD, Chenoweth SF, Doughty P, Blows MW (2005). Divergent selection and the evolution of signal traits and mating preferences. PLOS Biol 3: 1988-1995.

Sanjuan R, Nebot MR (2008). A network model for the correlation between epistasis and genomic complexity. PLOS ONE 3: e2663.

Segre D, Deluna A, Church GM, Kishony R (2005). Modular epistasis in yeast metabolism. Nat Genet 37: 77-83 
Sharp NP, Agrawal AF (2008). Mating density and the strength of sexual selection against deleterious alleles in Drosophila melanogaster. Evolution 62: 857-867.

Siller S (2001). Sexual selection and the maintenance of sex. Nature 411: 689-692.

Szafraniec K, Borts RH, Korona R (2001). Environmental stress and mutational load in diploid strains of the yeast Saccharomyces cerevisiae. Proc Natl Acad Sci USA 98: 1107-1112.

Szathmary E (1993). Do deleterious mutations act synergistically? Metabolic control theory provides a partial answer. Genetics 133: 127-132.

Uyenoyama MK (1993). Ecological and genetic factors directing the evolution of selffertilization. Oxf Surv Evol Biol 9: 327-381.

Wang AD, Sharp NP, Spencer CC, Tedman-Aucoin K, Agrawal AF (2009). Selection, epistasis, and parent-of-origin effects on deleterious mutations across environments in Drosophila melanogaster. Am Nat 174: 863-874.
Whitlock MC (2000). Fixation of new alleles and the extinction of small populations: drift load, beneficial alleles, and sexual selection. Evolution 54: 1855-1861.

Whitlock MC, Agrawal AF (2009). Purging the genome with sexual selection: reducing mutation load through selection on males. Evolution 63: 569-582.

Whitlock MC, Bourguet D (2000). Factors affecting the genetic load in Drosophila: synergistic epistasis and correlations among fitness components. Evolution 54: 1654-1660.

Yang HP, Tanikawa AY, Van Voorhies WA, Silva JC, Kondrashov AS (2001). Whole-genome effects of ethyl methanesulfonate-induced mutation on nine quantitative traits in outbred Drosophila melanogaster. Genetics 157: 1257-1265.

Young JA, Yourth CP, Agrawal AF (2009). The effect of pathogens on selection against deleterious mutations in Drosophila melanogaster. J Evol Biol 22: 2125-2129. 